\title{
Effect of fast-track surgery in gynecological laparoscopy: a randomized controlled trial ${ }^{\dagger}$
}

Xue-Ping Zhu, Sha-Sha Zhao*, Jie-Dan Qin

The Affiliated Wuxi Maternity and Child Health Care Hospital of Nanjing Medical University, Wuxi, Jiangsu 214000, China

Received: 23 October 2019; Accepted: 17 November 2019; Published: 20 September 2020

Abstract: Objective: To evaluate the value of fast-track surgery (FTS) in gynecological laparoscopy during the perioperative period.

Methods: The clinical data of 234 patients who underwent gynecological laparoscopy from January to December in 2018 were collected. Of note, 117 patients received a routine nursing method (control group), and 117 patients received the FTS nursing method (observation group).

Results: Compared with the control group, FTS group had a quicker postoperative bowel function recovery, earlier to get out of bed, and a lower anxiety level $(P<0.05)$; The incidence of postoperative nausea and vomiting of two groups had no statistical difference. Conclusions: The application of FTS in gynecological laparoscopy is safe, effective, and worth promoting.

Keywords: fast-track surgery • perioperative period $\bullet$ anxiety $・$ laparoscopy $・$ gynecological $\bullet$ postoperative

(c) Shanxi Medical Periodical Press.

\section{Introduction}

The concept of fast-track surgery (FTS) was first proposed by Danish Surgeon Kehlet in 1997. By applying perioperative optimization measures with evidencebased interventions, the incidence of postoperative complications reduced; at the same time, it reduces the hospital stay of patients to accelerate the postoperative rehabilitation of patients. ${ }^{1}$ Laparoscopic technology began in Europe in the 19th century. With the development and promotion of this technology, it is widely used in clinical departments. Compared with laparotomy, it has many advantages such as small wounds, short operation time, less intraoperative blood loss, and less pain. ${ }^{2}$ It is widely used in gynecology. In this study, we report the results of a randomized controlled trial of the FTS program on gynecological laparoscopic perioperative patients to evaluate the FTS protocol as an alternative method to better promote postoperative rehabilitation.

\footnotetext{
$\dagger \quad$ This project was supported by the effect of individualized preoperative intestinal preparation scheme on preoperative intestinal cleanliness in patients with cervical cancer (No. Q201607).
}

How to cite this article: Zhu XP, Zhao SS, Qin JD. Effect of fast-track surgery in gynecological laparoscopy: a randomized controlled trial. Front Nurs. 2020;3:235-238. 


\section{Materials and methods}

\subsection{Trial design and study subjects}

This study was a noninferiority, randomized controlled clinical trial involving gynecological laparoscopic patients. The study subjects were all enrolled patients who underwent laparoscopic surgery for gynecological diseases in our hospital from January 8 to December 20,2018 . A total of 234 patients enrolled were adults (aged between $\geq 18$ and $<70$ years). All participants or their guardians provided written informed consent and assent when they were eligible for the trial.

Participants were required to have a clear consciousness with no mental disorder, and they can fill in or answer the questionnaire content independently or with the help of the researcher.

Exclusion criteria were malignant changes, conversion to open surgery, severe psychological or neurological disease, or overlapping with other clinical trials: pregnancy, uncontrolled cardiopulmonary disease, comorbidities that could affect the quality of life, and nutritional status (e.g., liver cirrhosis and renal failure).

\subsection{Methods}

Eligible patients were randomly assigned to interventional and conventional management groups.

The conventional group, as a control group, underwent conventional, experience-based perioperative managements:

(1) Provide verbal education before surgery, explaining the relevant knowledge and precautions about the surgery;

(2) Assist patients in routine preoperative preparation: oral medication for bowel preparation even mechanical bowel preparation until watery stool; regardless of the time of the operation, all patients start fasting and water restriction at 10 p.m. the previous night;

(3) Provide a small amount of liquid food to the patients $6 \mathrm{~h}$ after surgery, then according to the patient's gastrointestinal function recovery transit to semiliquid, soft food, and general food gradually;

(4) Encourage patients to get out of bed early.

The interventional group, as a test group, with FST cares performed:

(1) Psychological care: Primary nurses evaluate the patient's psychological condition; guide them to maintain a good mood; encourage family members to communicate with patients; and provide family support.

(2) Health education: Developed a laparoscopic perioperative mind map, combined with the patient's education level, understanding ability, targeted use of verbal, card, demonstration, and other methods for health education. Included preoperative preparation, early postoperative activities, and postoperative complications.

(3) The patient does not receive mechanical bowel preparation and oral enteral cleansing agents before surgery, hence liquid stool does not occur.

(4) The patients should have fasting $6 \mathrm{~h}$ before surgery and stop drinking water $4 \mathrm{~h}$ before surgery.

(5) After returning to the room, the lower extremity gastrocnemius muscle massage was performed. After $6 \mathrm{~h}$, the patient was instructed to ankle pump exercise and turn over on the bed. After $6 \mathrm{~h}$, the patient was assisted and guided to stand by the bed. The patient was allowed to get out of bed $12 \mathrm{~h}$ after surgery.

(6) The patients are allowed to eat porridge soup $4 \mathrm{~h}$ after surgery, and if there is no discomfort, then the patients are allowed to restore the general food $12 \mathrm{~h}$ after surgery;

(7) The patients should be treated effectively with discomforts such as pain, nausea, and vomiting.

Since all subjects who were randomized and received any study intervention were obliged to follow the study protocol and monitored for best compliance, the per-protocol set or safety set was not defined differently. Neither patients nor their caregivers were masked to the allocation assignments, but adjudicators of outcome were masked.

\subsection{Observation index}

(1) Postoperative rehabilitation index: The incidence of postoperative anal exsufflation time, postoperative activity time, and postoperative nausea and vomiting (PONV) were observed. The aim was to determine whether the FTS protocol was not inferior to conventional management.

(2) Anxiety status of patients during the perioperative period: Self-rating Anxiety Scale (SAS), a method of measuring levels of anxiety in patients who have anxiety-related symptoms, was used. ${ }^{3}$ The test uses a 4-point Likert scale for the responses, ranging from 1 (none of the time) to 4 (most of the time). The form of the questionnaire is used; the contents of the questionnaire are printed uniformly; and unified 
terminology is used in the process of relevant guidance. After completion, the questionnaire is collected.

\subsection{Statistical analysis}

All the statistical analyses were performed with SPSS 20.0. All data analysis was performed according to a pre-established analysis plan. A two-sided 95\% confidence interval for the difference was calculated to test for preponderance. Categorical variables or proportions were compared by using $\chi^{2}$ tests with continuity correction or Fisher's exact test when appropriate. Continuous variables were compared using Student's $t$-test. The tests of significance were undertaken at the two-sided $\alpha$-level of 0.05 .

\section{Results}

\subsection{Patients' characteristics}

From January 8 to December 20, 2018, a total of 266 consecutive patients were assessed for eligibility for this study, and 234 patients underwent randomization. Of note, 32 patients were excluded because of changing surgical plans during operation (7), refusing study participation after allocation (14), or refusing operation after allocation (11) and 117 patients in each group started the allocated perioperative management. The demographics and preoperative data were similar in both study groups (Table 1).

\subsection{Study outcomes}

In this study, the primary outcome is that the anal exhaust recovery time in the FTS group was $19.64 \pm 6.85$ and that of the conventional group was $22.67 \pm 9.08$. In contrast, anal exhaust time in the control group was earlier, and the difference was statistically significant $(P=0.007)$. On the other hand, the postoperative activity lasted $22.61 \pm 6.55 \mathrm{~min}$ in the FTS group, in contrast with the conventional group which lasted $20.09 \pm 8.82 \mathrm{~min}$, and it is much longer and also had statistical difference $(P=0.018)$.

The secondary outcome is the incidence of PONV in the two groups: four in the FTS group and nine in the conventional group. There was no significant difference between the two groups $(P>0.05)$.

The third outcome is that the anxiety level of patients in the FTS group was $49.09 \pm 7.11$, which was significantly lower than that in the conventional group after surgery $(P=0)$.

These results also showed the noninferiority of the FTS protocol with conventional management even more preponderance (Table 2).

\section{Discussion}

With the development of minimally invasive surgery, laparoscopic surgery has currently become an important way to treat gynecological diseases. It is easy to cause adverse reactions such as nervousness and anxiety, since laparoscopic surgery itself is a source of stress, especially combined with insufficient relevant knowledge, more importantly, female genitalia always involved in gynecological surgery. ${ }^{4}$ Studies have shown that the incidence of anxiety is at a fairly high level of $69 \%$ in patients in the gynecological laparoscopic perioperative period. When this state of anxiety continues, the resistance and immunity of the patient will be reduced which in turn reduce the patient's tolerance to surgery, increase the intraoperative complications and the incidence of postoperative infection, and affect the

\begin{tabular}{lcc}
\hline Characteristics & FTS $(n=117)$ & Conventional $(n=117)$ \\
\hline \hline Age, years & $38.73 \pm 9.64$ & $39.79 \pm 10.44$ \\
Comorbidity, $n(\%)$ & & \\
Hypertension & $8(6.8)$ & $9(7.7)$ \\
Diabetes & $1(0.9)$ & $2(17.1)$ \\
\hline
\end{tabular}

Note: Continuous variables are expressed with means \pm standard deviations.

FTS, fast-track surgery.

Table 1. Baseline clinical characteristics of the patients

\begin{tabular}{|c|c|c|c|c|c|}
\hline Group & Number & $\begin{array}{c}\text { Anal exhaust } \\
\text { recovery time (h) }\end{array}$ & $\begin{array}{c}\text { Postoperative } \\
\text { activity time (min) }\end{array}$ & $\begin{array}{l}\text { Incidence of } \\
\text { PONV, } n(\%)\end{array}$ & $\begin{array}{l}\text { SAS anxiety } \\
\text { score }\end{array}$ \\
\hline Conventional group & 117 & $22.67 \pm 9.08$ & $20.09 \pm 8.82$ & $9(7.7 \%)$ & $56.32 \pm 7.97$ \\
\hline FTS group & 117 & $19.64 \pm 6.85$ & $22.61 \pm 6.55$ & $4(3.4 \%)$ & $49.09 \pm 7.11$ \\
\hline$t$ & & 2.87 & 2.47 & & 7.30 \\
\hline$\chi^{2}$ & & & & 2.04 & \\
\hline$P$ & & 0.007 & 0.018 & 0.15 & 0 \\
\hline
\end{tabular}

PONV, postoperative nausea and vomiting.

Table 2. Study outcomes in two groups of patients. 
recovery. ${ }^{5}$ Therefore, we must pay more attention to the psychological problems of gynecological laparoscopic perioperative patients. Meanwhile, support from family members would help patients to overcome the obstacle.

FTS aims to improve, optimize, and combine the health care behaviors that affect the normal physiological state and increase the stress response of patients during the perioperative period to minimize the body stress response and promote rapid recovery after surgery. There is evidence in the literature showing that FTS is beneficial in the management of patients by improving the quality of care, and it has been widely recognized, especially in Western countries.

Consensus has been built in the application of FTS in the colorectal perioperative period ${ }^{6}$ similar to the gynecological laparoscopy. The FTS concept gives full play to the human-oriented concept of nursing care, and it takes patients as the center to promote patient recovery.

In this study, the observation group was given conventional care plus the FTS concept. The results showed that the interventions from the preoperative and postoperative factors influencing the rapid recovery of the patients may effectively reduce the anal exhaust recovery time, increase the duration of postoperative activity time, and improve the patients' comfort. In addition, assessing the patients' anxiety level through appropriate psychological counseling and improving the patients' perioperative awareness and other countermeasures have a positive impact on their psychological state, which may result in better patient coordination, improve patients' quality of life, and promote their postoperative rapid rehabilitation.

This study also showed that the FTS concept has no statistically significant difference in the incidence of PONV compared with the traditional concept of gastrointestinal preparation. Also, the percentage of occurrence

\section{References}

1. Kehlet $H$, Wilmore DW. Evidence-based surgical care and the evolution of fast-track surgery. Ann Surg. 2008;248:89-98.

2. Li P, Li CY. Clinical application decision of laparoscopic surgery for uterine fibroids. J Appl Gynecol Endocrinol (electronic version). 2017;4:18-20 (in Chinese).

3. Duan $Q Q$, Sheng L. Clinical validity of the self-rating scale for anxiety and depression. Chin J Mental Health. 2012;26:676-679 (in Chinese).

4. Gao F, Zhang LQ, Sun BB. Investigation of preoperative anxiety in patients undergoing gynecological laparoscopic surgery. PLA J Nurs. 2014;31:73-74 (in Chinese). is even lower than the traditional model. The increased probability of PONV from the traditional model may be related to dehydration caused by prolonged preoperative fasting time and premature bowel preparation time to avoid pulmonary aspiration caused by endotracheal intubation. ${ }^{7}$ The FTS advocates minimizing preoperative mechanical enema and reducing irritation to the gastrointestinal tract.

There are several limitations to our trial. First, to strengthen internal validity and reduce heterogeneity, the study population was restricted only to patients who received gynecological laparoscopy without malignant change. This restriction might limit generalizability. Second, a masking process was not fully applied in this study. Specifically, patients and caregivers were not masked to treatment assignments because masking in these groups was impossible. Finally, this study was conducted in a single center.

\section{Conclusions}

We believe that the FTS protocol is effective in gynecological laparoscopic perioperative care, and it is worth promoting. Systematic efforts are needed for active diffusion of the enhanced recovery after surgery (ERAS) in the perioperative care model and they should be considered the standard of care in gynecological surgery.

\section{Ethical approval}

Ethical issues are not involved in this article.

\section{Conflicts of interest}

All contributing authors declare no conflicts of interest.
5. Chu CM, Xue XL, Cai YH. Analysis of influencing factors of anxiety in gynecological perioperative patients and effect of precision nursing intervention. Fam Plann Obstetrics Gynecol China. 2018; 10:37-40 (in Chinese).

6. Lassen K, Soop M, Nygren J, et al. Consensus review of optimal perioperative care in colorectal surgery: Enhanced Recovery After Surgery (ERAS) Group recommendations. Arch Surg. 2009;144:961-969.

7. Song Y, Li Y, Zheng WH, Dong YJ. Research progress on prevention and treatment of postoperative nausea and vomiting. Pract Med Clin. 2017; 20:720-724 (in Chinese). 